\title{
CARRIER RESHAPING AND MUX-DEMUX FILTERING IN 0.8 BIT/S/HZ WDM RZ-DPSK TRANSMISSION
}

\author{
Ranjeet S Bhamber, ${ }^{1}$ Sergei K Turitsyn, ${ }^{1}$ and Vladimir Mezentsev ${ }^{1}$ \\ ${ }^{1}$ Aston University, Bitmingham B4 7ET, United Kingdom
}

\begin{abstract}
Numerical optimization of the ultra high dense WDM RZ-DPSK transmission has been performed by means of fine tuning the duty cycle and shape, carrier reshaping, and pre- and post-filtering parameters.
\end{abstract}

\section{INTRODUCTION}

High spectral efficiency is an ultimate target in modern long haul fiber communications. New signal formats and RZ-DPSK in particular have become key enabling factors in increasing spectral efficiency [1-3]. However, experimental studies of applications of DPSK format in optical transmission have recently outpaced theoretical and numerical analysis. Such important resources for reaching the highest system performance as carrier reshaping and channel pre- and post-filtering have not been systematically studied in case of new formats. Asymmetric pre-filtering has been thoroughly studied for on-off keying formats, see e.g. [4] and has recently been used for CS-RZ DPSK [5].

In this paper we have systematically explored the optimal duty cycle, carrier reshaping and channel pre- and post- filtering on FEC free system performance by means of numerical optimization. Our main purpose is to fine tune the system and signal parameters corresponding to the highest experimentally available transoceanic transmission at spectral efficiency of $0.8 \mathrm{bit} / \mathrm{s} / \mathrm{Hz}$ recently reported in [6].

\section{SYSTEM CONFIGURATION}

The chosen system is essentially equivalent to that reported in [6]. 


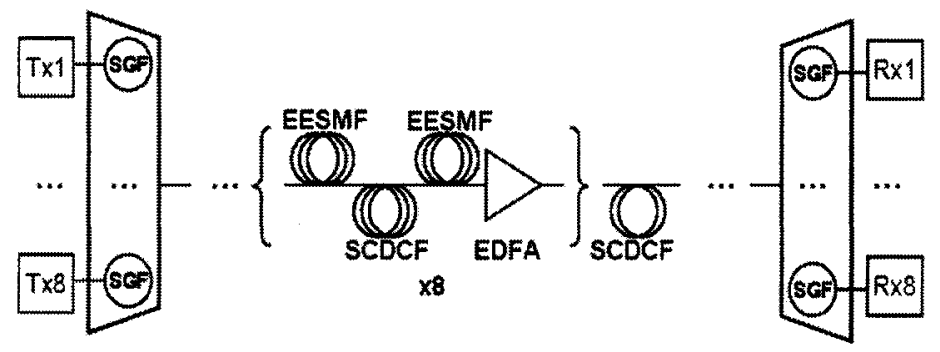

Figure 1. Scheme of the modelled system. The same super-Gaussian filters (SGF) are used for pre- and post-filtering.

The transmission line comprises periodically allocated dispersion map including eight symmetrically deployed pairs of Enlarged Effective area SMF (EESMF) with slope compensating DCF (SCDCF) between the spans of EESMF. Fiber parameters are summarized in Table 1 and the scheme of the modelled system is shown in Fig 1.

Each symmetric map is followed by EDFA to compensate for losses. The last section is followed by the short span of $3.75 \mathrm{~km}$ of SCDCF to equalize the residual chromatic dispersion over eight symmetric sections.

Table 1. Fibre parameters

\begin{tabular}{lcc}
\hline Fibre & EESMF & SCDCF \\
\hline Dispersion, $p s / n m / k m$ & 20 & -40 \\
Dispersion slope, $p s / \mathrm{nm}^{2} / \mathrm{km}$ & 0.06 & -0.12 \\
Effective area $\mu m^{2}$ & 110 & 30 \\
Length per section, $\mathrm{km}$ & 28 & 15 \\
\hline
\end{tabular}

Following [6], the choice of simple components was made to demonstrate superior ultra high density, up to $80 \%$ spectral efficient, transmission by means of fine tuning of system and signal parameters. No polarization division multiplexing have been used that could be considered as additional resort to improve the presented results.

\section{MODELLING}

We have performed numerical modelling of 8 channel RZ-DPSK transmission. Each channel had been pre-filtered before multiplexing in order to reduce inter-channel cross-talk. The same filters were used in demultiplexor. The following parameters were tuned during the optimization: i) MUX/DEMUX filter detuning, bandwidth, and shape. Super-Gaussian filters of different order with 

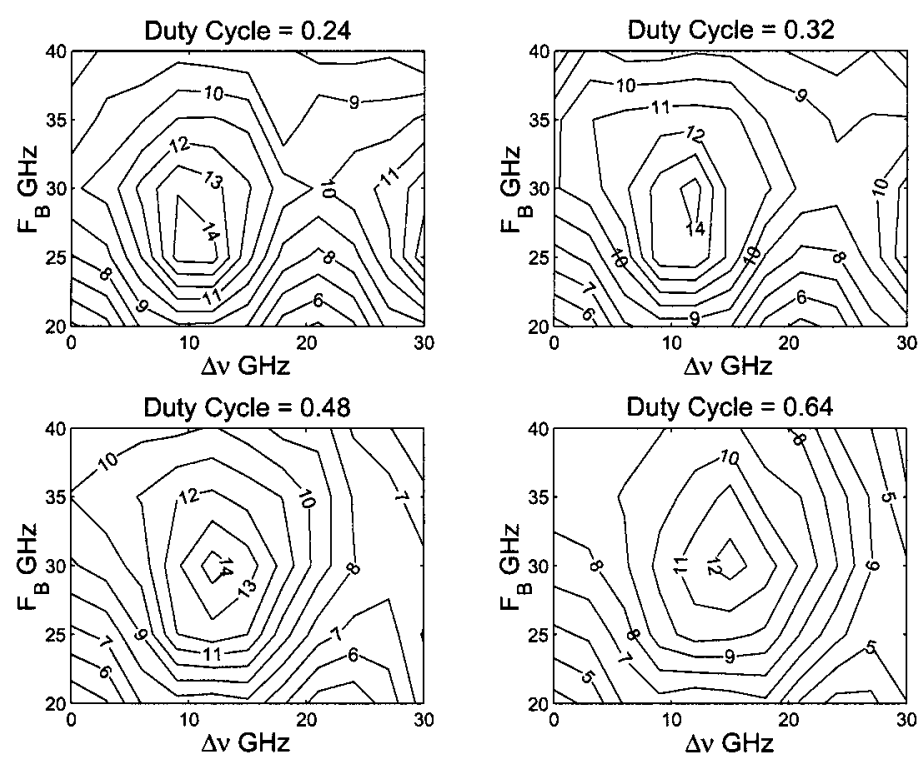

Figure 2. Contour plots of linear Q-factor versus filter bandwidth and detuning for different duty cycles.

filter transfer function $H(f)=\exp \left\{-\left[\left(f-f_{0}\right) / B\right]^{N / 2}\right\}$ have been tried to find the optimal filter steepness parameter $N$ as well as filter bandwidth $B$ and detuning $f_{0}$. ii) carrier duty cycle and carrier shapes varied from Gaussian to super-Gaussian of different orders.

A multi-stage optimization strategy has been adopted due to a very large number of optimization parameters. First, back to back optimization has been performed of the carrier duty cycle and shape as well as pre- and post-filtering parameters.

\section{RESULTS}

The results of this optimization are presented in Figures 2,3 and 4. Fig. 2 shows the contours of the linear Q-factor versus filtering parameters (filter bandwidth and detuning) for different pulsewidth. Filter shapes were kept Gaussian. It was found that pulsewidth of $6 \mathrm{ps}$ provides the best performance.

Fig 3 shows the contours of the linear Q-factor versus the same filter parameters but for different filter shapes for the fixed duty cycle. It was found that steeper filters provide better discrimination between the channels and practically eliminate inter-channel crosstalk from $\mathrm{N}=12$. In all the following simu- 

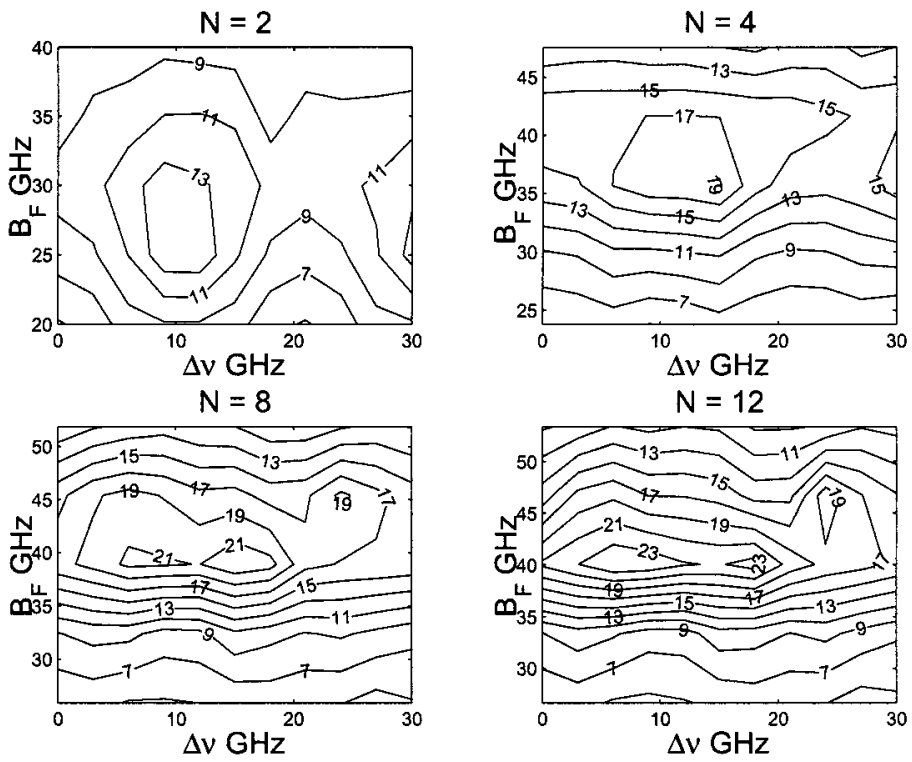

Figure 3. Contour plots of linear q-factor versus filter bandwidth and detuning for different filter shapes: Gaussian: (a) $\mathrm{N}=2$ and Super-Gaussian (b) $\mathrm{N}=4$, (c) $\mathrm{N}=8$, (d) $\mathrm{N}=12$.

lations this parameter was set to 16 as steeper filters do not gain performance and could be difficult to manufacture.

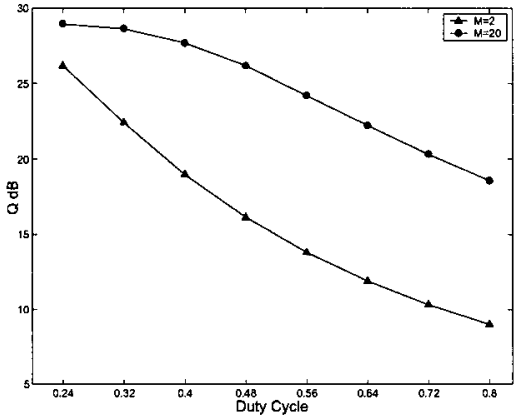

Figure 4. Back to back Q-Factor versus duty cycle for different carrier shapes: Gaussian $\mathrm{M}=2$ (triangles) and super-Gaussian $M=20$ (circles)

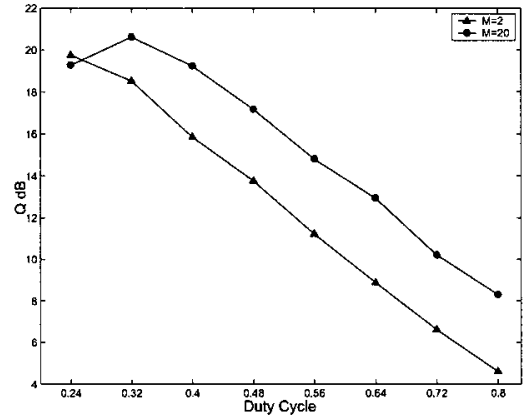

Figure 5. Q-Factor (after $2000 \mathrm{~km}$ ) versus duty cycle for different carrier shapes: Gaussian $\mathrm{M}=2$ (triangles) and super-Gaussian $M=20$ (circles) 
Figures 4 and 5 shows the influence of the carrier shape on system performance. Fig 4 shows back to back performance gain by using a super-Gaussian carrier versus duty cycle. It is seen that the performance gain achieved by simply reshaping the carrier can be as much as $10 \mathrm{~dB}$ whereas after $2000 \mathrm{~km}$ the performance gain is still respectable $4 \mathrm{~dB}$ in a wide range of the duty cycle as shown in Fig 5.

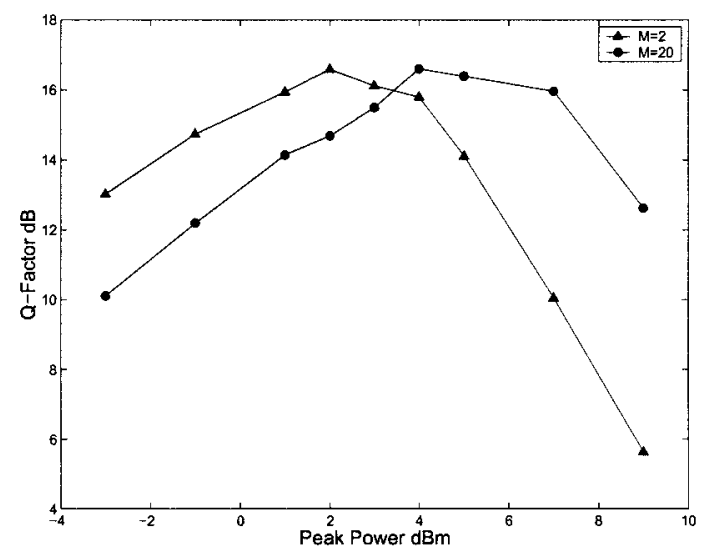

Figure 6. Q-Factor versus signal peak power cycle for different carriers: $\mathrm{M}=2$ (triangles) and $\mathrm{M}=20$ (circles) after $2000 \mathrm{~km}$.

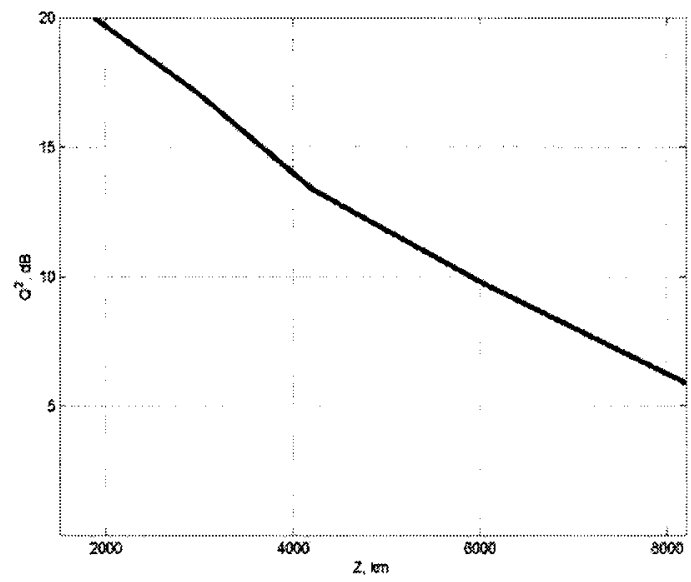

Figure 7. Q-Factor versus transmission distance for optimized transmission.

Figure 6 illustrates another benefit of using super-Gaussian carrier with steep shape index $M=20$. It shows the system performance after $2000 \mathrm{~km}$ ver- 
sus signal peak power. It is seen that super-Gaussian signals perform better at higher power by approximately $2 \mathrm{~dB}$ which results in a noticeably better signal to noise ratio.

\section{CONCLUSIONS}

System performance at $0.8 \mathrm{bit} / \mathrm{s} / \mathrm{Hz}$ CS-RZ DPSK transmission have been systematically explored and optimized by fine tuning of duty cycle, carrier reshaping, and pre- and post filtering. It was found that ultra high density system gains performance by using super-Gaussian carriers with relatively short duty cycle of 0.24 and steep super-Gaussian filters to reduce inter-channel crosstalk.

\section{REFERENCES}

[1] J.-X. Cai et al., OFC 2004, PD34 (2004)

[2] A. H. Gnauck et al., OFC 2004, PD35 (2004)

[3] B. Zhu, OFC 2004, ThEl (2004)

[4] A. Agata et al., OFC 2003, MF78 (2003)

[5] K. Tanaka, I.Morita, and N.Edagawa, OFC 2004, TuF2 (2004)

[6] I. Morita and N. Edagawa, ECOC2003, PD (2003) 